\title{
Index to genera and species, Volume 15 (2004)
}

Abacoproeces saltuum (Koch)..........................156, 189

Abdera affinis (Paykull) .......................................... 6-22

Abdera flexuosa (Paykull) ........................................ 6-22

Absidia schoenherri Dejean ................................. 72-90

Acanthaclisis occitanica (Villers) .......................221, 223

Acantholycosa lignaria (Clerck)........................173, 191

Acanthothrips nodicornis (Reuter) ......................... 72-90

Acartophthalmus nigrinus (Zetterstedt) .................. 72-90

Acer mono .............................................................. 130

Acompocoris alpinus Reuter................................ 72-90

Acompocoris pygmaeus (Fallén) ............................ 72-90

Acrostiba borealis Thomson................................... 72-90

Acrulia inflata (Gyllenhal)................................ 6-22, 110

Actia resinellae (Schrank) ................................... 72-90

Aculepeira packardi (Thorell)............................. 115-116

Adoristes ovatus (Koch) ....................................... 72-90

Adoristes poppei (Oudemans) ................................ 72-90

Adscita statices Linnaeus ...................................... 91-101

Aeolothrips vittatus Haliday ................................. 72-90

Agaricochara latissima (Stephens).......................... 6-22

Agathidium confusum Brisout de Barneville..............6-22

Agathidium laevigatum Erichson............................... 6-22

Agathidium nigripenne (Fabricius) .......................... 6-22

Agathidium rotundatum (Gyllenhal) ........................ 6-22

Agathidium seminulum (Linnaeus) .......................... 6-22

Aglais urticae (Linnaeus) ..................................... 34-40

Agnyphantes expunctus (P-Cambridge)....72-90, 156, 189

Agrilus angustulus (Illiger)................................... 57-59

Agroeca brunnea (Blackwall) .....................172, 184, 191

Agroeca proxima (P-Cambridge) .........172-173, 184, 191

Aguriahana germari (Zetterstedt)........................... 72-90

Agyneta affinisoides (Tanasevitch) ................................ 116

Agyneta birulai (Kulczynski) ..................................... 115

Agyneta cauta (P-Cambridge) ........................... 156, 189

Agyneta conigera (P-Cambridge).................156-157, 189

Agyneta olivacea (Emerton) .............................. 157, 189

Agyneta ramosa Jackson .................................... 157, 189

Agyneta rurestris ................................................... 163

Agyneta subtilis (P-Cambridge) .........................157, 189

Aleochara moerens Gyllenhal ................................ 6-22

Aleochara sanguinea (Linnaeus) .............................. 6-22

Allacma fusca (Linnaeus) ...................................... 72-90

Alliopsis brunneigena (Schnabl).............................72-90

Alliopsis denticauda (Zetterstedt) ........................... 72-90

Alliopsis silvestris (Fallén) .................................... 72-90

Allium schoenoprasum .................................................. 161

Allomengea scopigera (Grube) ....................157, 184, 189

Alluaudomyia quadripunctata (Goetghebuer)..........72-90

Alnus..................................... 166, 170, 172, 174-176

Alnus glutinosa...

173,191

Alopecosa aculeata (Clerck).

$115-116$
Alopecosa borea (Kulczynski) ............................115-116

Alopecosa inquilina (Clerck)............................. 173, 191

Alopecosa kulczynski Sternbergs ................................. 116

Alopecosa pinetorum (Thorell) ............................ 173, 191

Alopecosa pulverulenta (Clerck) .........................173, 191

Alopecosa sibirica (Kulczynski).................................. 116

Alopecosa taeniata (Koch) .........................173-174, 191

Altica deserticola (Weise) ............................................ 1-5

Altica lencorana Konstantinov ............................ 138-141

Altica longicollis (Allard) ................................... 138-141

Altica oleraea (Linnaeus).......................................... 1-5

Ampedus balteatus (Linnaeus)................................ 72-90

Ampedus nigrinus (Herbst).................................... 72-90

Amphicyllis globus (Fabricius) ................................. 6-22

Amylocystis lapponica............................................ 110

Anachyra van Achterberg ........................................ 225

Anaspis arctica Zetterstedt ................................... 6-22

Anaspis marginicollis Lindberg ..................... 6-22, 72-90

Anaspis rufilabris Gyllenhal............................... 72-90

Anchioleucopis geniculata (Zetterstedt)..................72-90

Anguliphantes engulipalpis (Westring) ................157, 189

Anisotoma axillaris Gyllenhal ................................. 6-22

Anisotoma castanea (Herbst) .................................. 6-22

Anisotoma glabra (Kugelann) ................................. 6-22

Anisotoma humeralis (Fabricius) ..................6-22, 72-90

Anopleta corvina (Thomson)................................... 6-22

Anoplus plantaris (Naezen) ................................... 72-90

Anotylus nitidulus (Gravenhorst) ............................. 6-22

Anthocoris nemorum (Linnaeus) ........................... 72-90

Anthomyia mimetica (Malloch) ........................... 72-90

Anthonomus phyllocola (Herbst) ............................ 72-90

Antophagus omalinus Zetterstedt........................... 72-90

Antrodia serialis .......................................................... 7

Anurophorus laricis Nicolet ................................... 72-90

Anurophorus septentrionalis Palissa.......................72-90

Aphidecta obliterata (Linnaeus) ............................. 72-90

Aphthona atrocaerulae (Stephens)........................ 138-141

Aphthona flava Guillebeau........................................ 140

Aphthona kocheri Kral ............................................... 139

Aphthona nigriscutis Foudras...................................... 140

Aphthona violaceae (Koch) ................................. 138-141

Aplocnemus nigricornis (Fabricius)........................ 72-90

Apotomopterus........................................................... 130

Apteromyia claviventris (Strobl) ............................ 72-90

Aptinothrips stylifer Trybom ............................... 72-90

Araneus diadematus Clerck................................ 151, 188

Araneus nordmanni (Thorell) ............................ 151, 188

Araneus omoedus ...................................................... 151

Araneus quadratus Clerck ................................ 151, 188

Araneus sturmi (Hahn) .................................... 151, 188

Araniella displicata (Hentz)............................... 115-116

Archinemapogon yildizae Kocak ................................. 107 
Arctella lapponica (Holm)..................................... 116 Arianella cf. cucurbitina (Clerck)........................... 72-90 Aricia artaxerxes (Fabricius)....................................... 101 Asagena phalerata .................................................. 181

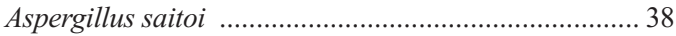
Aspidiphorus orbiculatus (Gyllenhal)...................... 6-22 Asthenargus paganus (Simon)...........................157, 189 Atea sturmi (Hahn) ....................................... 72-90, 151 Atheta (Alaobia) gagatina (Baudi) ........................... 6-22 Atheta (Alaobia) pallidicornis (Thomson) ................. 6-22 Atheta (Alaobia) sodalis (Erichson)......................... 6-22 Atheta (Datomicra) celata (Erichson)....................... 6-22 Atheta (Datomicra) dadopora Thomson................... 6-22 Atheta (Datomicra) nigra (Kraatz) .......................... 6-22 Atheta (Dimetrota) aeneipennis (Thomson)...............6-22 Atheta (Microdota) pittionii Scheerpeltz................... 6-22 Atheta (Microdota) subtilis (Scriba) ......................... 6-22 Atheta (Mocyta) fungi (Gravenhorst)........................ 6-22 Atheta (Mycetota) laticollis (Stephens) ...................... 6-22 Atheta (Plataraea) nigritula (Gravenhorst)............... 6-22 Atheta (s. str.) boletophila (Thomson) ....................... 6-22 Atheta (s. str.) britanniae Bernhauer \& Scheerpeltz ...6-22 Atheta (s. str.) castanoptera (Mannerheim)...............6-22 Atheta (s. str.) crassicornis (Fabricius) ..................... 6-22 Atheta (s. str.) diversa (Sharp) ................................ 6-22 Atheta (s. str.) euryptera (Stephens)......................... 6-22 Atheta (s. str.) fungicola (Thomson) ......................... 6-22 Atheta (s. str.) harwoodi Williams ............................. 6-22 Atheta (s. str.) liturata (Stephens) ............................. 6-22 Atheta (s. str.) nigricornis (Thomson)....................... 6-22 Atheta (s. str.) oblita (Erichson) ................................ 6-22 Atheta (s. str.) paracrassicornis Brundin ................... 6-22 Atheta (s. str.) pilicornis (Thomson) ......................... 6-22 Atheta (s. str.) ravilla (Erichson)............................... 6-22 Atheta (Traumoecia) picipes (Thomson) ................... 6-22 Atheta picipennis Mannerheim ............................. 72-90 Athous vittatus (Fabricius).................................... 72-90 Atomaria affinis (Sahlberg) .................................... 6-22 Atomaria alpina Heer ........................................... 6-22 Atomaria apicalis Erichson...................................... 6-22 Atomaria fuscata (Schönherr) .................................... 6-22 Atomaria pusilla (Paykull) ...................................... 6-22 Atomaria turgida Erichson ..................................... 72-90 Atrichopogon lucorum (Meigen) .......................... 72-90 Atrichopogon minutus (Meigen)............................. 72-90 Austrolimnophila (Archilimnophila) unica

(Osten-Sacken)... .. 47

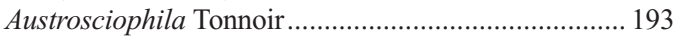

Autalia longicornis Scheerpeltz.............................. 6-22

Bathyphantes gracilis (Blackwall)....

Bathyphantes nigrinus (Westring).

157,189

Bathyphantes parvulus (Westring)......................158, 189

Bathystomus Foerster ............................................. 225

Belba compta (Kulczynski) .................................. 72-90

Betula ........................................... 55, 59, 105, 150

Betula dahurica ..................................................... 130

Betula pendula .................................................... 7, 199

Betula pubescens....................................... 7, 67, 199

Bezzia affinis (Staeger) ......................................... 72-90

Bezzia bicolor (Meigen) ....................................... 72-90
Bezzia nigritula (Zetterstedt) ................................. 72-90

Bezzia rhynchostylata Remm ................................ 72-90

Bezzia solstitialis (Winnertz) ................................. 72-90

Bianor aurocinctus........................................ 178-179

Bibio nigriventris Haliday .................................... 72-90

Bibloporus bicolor (Denny) ................................. 72-90

Bicellaria austriaca Tuomikoski ........................... 72-90

Bicellaria nigra (Meigen) ..................................... 72-90

Bicellaria pilosa Lundbeck .................................. 72-90

Bicyclus anynana (Butler)........................................... 37

Bitoma crenata (Fabricius).................................... 6-22

Bjerkandera adusta .................................................... 7

Blepharomyia pagana (Meigen) ........................... 72-90

Bolethyphantes index (Thorell).........................158, 189

Boletina atridentata sp. n. ..................................23-33

Boletina bidenticulata Sasakawa \& Kimura ..................26

Boletina cordata sp. n. .......................................... 23-33

Boletina dispecta Dziedzicki.......................................25

Boletina falcata sp. n............................................ 23-33

Boletina fennoscandiaca sp. n.............................23-33

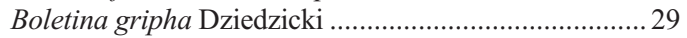

Boletina hedstroemi sp n. ....................................23-33

Boletina kivachiana sp. n. ................................... 23-33

Boletina lapponica sp. n.....................................23-33

Boletina lundbecki Lundström ..................................... 32

Boletina nigricans Dziedzicki ...................................... 31

Boletina nigrofusca Dziedzicki .................................... 28

Boletina nitida Grzegorzek .......................................... 25

Boletina sciarina .................................................. 27-28

Boletina silvatica Dziedzicki....................................... 30

Boletina struthioides sp. n. ..................................23-33

Boletina subtriangularis sp. n..............................23-33

Boletina triangularus Polevoi ...................................... 30

Bolitochara lucida (Gravenhorst)........................... 6-22

Bolitochara mulsanti Sharp .................................... 6-22

Bolitochara obliqua Erichson.................................... 6-22

Bolitochara pulchra (Gravenhorst) ......................... 6-22

Bolitophagus reticulatus (Linnaeus) ......................... 6-22

Bolyphantes alticeps (Sundevall) ................158, 184, 189

Bolyphantes crucifer (Menge) ............................... 72-90

Bolyphantes index................................................. 158

Bolyphantes luteolus (Blackwall) ...................... 158, 189

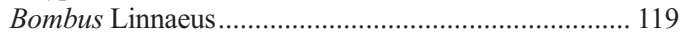

Borboropsis puberula (Zetterstedt).........................72-90

Bourletiella pistillum Gisin ................................... 72-90

Brachonyx pineti (Paykull)..................................... 72-90

Brachypogon bialoviesicus Krzywinski.................. 72-90

Brachypogon incompletus (Kieffer).......................72-90

Brachypogon nitidulus (Edwards) ......................... 72-90

Brachypogon norvegicus Szadziewski \& Hagan......72-90

Brachypogon perpusillus (Edwards)...................... 72-90

Brachypogon sociabilis (Goetghebuer)...................72-90

Brachypogon vitiosus (Winnertz) ......................... 72-90

Bradysia brevispina Tuomikoski ........................... 72-90

Bryaxis puncticollis (Denny) .................................. 72-90

Bryimyia gibbosa (Felt)....................................... 72-90

Bubopsis hamata Klug ........................................... 222

Bubopsis zarudnyi Alexandrova-Martynova................222

Caenopachyella Szépligeti........................................ 225

Caenoscelis ferruginea (Sahlberg) ............................. 6-22 
Caledonia evansi ................................................ 167 Calluna....................... 151, 165, 171-174, 176, 178, 180 Calystegia sepium.................................................... 50 Camisia biurus (Koch) ......................................... 72-90 Camisia segnis (Hermann) .................................... 72-90 Camponotus herculeanus (Linnaeus).......................72-90 Camptochaeta camptochaeta (Tuomikoski) ............72-90 Camptochaeta hitula (Lengersdorf)........................ 72-90 Camptomyia Kieffer 107

Camptozygum aequale (Villers) ............................72-90

Carabodes labyrinthicus (Michael) .........................72-90

Carabodes ornatus Storkan.................................... 72-90

Carabodes willmanni Bernini................................72-90

Carabus brandti Faldermann........................... 129-137

Carabus canaliculatus Adams..........................129-137

Carabus crassesculptus Kraatz...........................129-137

Carabus granulatus Linnaeus ............................ 129-137

Carabus hummeli Fischer-Waldheim .................129-137

Carabus manifestus Kraatz............................... 129-137

Carabus sculptipennis Chaudoir........................129-137

Carabus smaragdinus Fischer ............................ 129-137

Carabus sui Imura \& Zhou.................................. 129-137

Carabus vladimirskyi Dejean ............................ 129-137

Carduus mutans ...................................................... 140

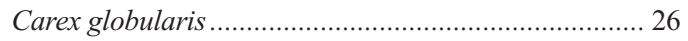

Carphacis striatus (Olivier) ..................................... 6-22

Carphoborus cholodkovskyi Spessivtseff.............198-210

Carphoborus minimus (Fabricius) ....................... 198-210

Carphoborus rossicus Semenov ......................... 198-210

Carphoborus teplouchovi Spessivtseff................198-210

Caryoscapha limbatum (Erichson) ........................... 6-22

Catops nigricans (Spence) ...................................... 6-22

Catops nigrita Erichson ......................................... 6-22

Cecidomyia pini (DeGeer) ................................... 72-90

Cedestis subfasciella (Stephens) .............................72-90

Celtis bungeana ............................................... 142-148

Centaurea glastifolia ...................................... 139-140

Centromerus arcanus (Cambridge) ..........72-90, 158, 189

Centromerus expertus ........................................... 168

Centromerus sylvaticus (Blackwall).............158, 184, 189

Cepheus cepheiformis (Nicolet) .............................. 72-90

Cepheus latus Koch ............................................. 72-90

Ceratinella brevis (Wider)...................158-159, 184, 189

Ceratinella scabrosa (P-Cambridge) ...................159, 189

Ceratinops pectinata (Tullgren) .................................. 170

Ceratopogon lacteipennis Zetterstedt ..................... 72-90

Ceratoppia bipilis (Hermann) ................................ 72-90

Ceratothrips ericae (Haliday) ............................... 72-90

Cercidia prominens (Westring)..........................151, 188

Cercyon impressus (Sturm) ...................................... 6-22

Cercyon lateralis (Marsham).................................... 6-22

Cerobasis guestfalica Kolbe................................... 72-90

Cerodontha (Poemyza) incisa (Meigen) ..................72-90

Cerrena unicolor ........................................................... 8

Cerylon deplanatum Gyllenhal................................ 6-22

Cerylon fagi Brisout de Barneville ........................... 6-22

Cerylon ferrugineum Stephens ............................... 6-22

Cerylon histeroides (Fabricius) ............................... 6-22

Chaetocnema breviuscula (Faldermann) ..................... 140

Chaetocnema concinna (Marsham) .............................. 140
Chaetocnema hortensis (Fourcroy) .......................... 140

Chaetocnema tibialis (Illiger)....................................... 140

Chamobates borealis (Trägårdh) ............................ 72-90

Chamobates pusillus Berlese.................................. 72-90

Chlorochroa pinicola (Mulsant \& Rey) ..................72-90

Choreutinula inermis (Tullberg) ............................. 72-90

Chromatomyia fuscula (Zetterstedt) ....................... 72-90

Chrysopa dubitans McLachlan ................................... 220

Chrysoperla carnea (Stephens) ................................... 220

Cis alter Silfverberg ............................... 6-22, 107-108

Cis bidentatus (Olivier)............................. 6-22, 102-112

Cis boleti (Scopoli) ................................................. 6-22

Cis castaneus Mellié ................................................ 110

Cis comptus Gyllenhal ......................................... 6-22

Cis fagi Waltl ........................................................ 110

Cis fissicornis Mellié............................................... 6-22

Cis glabratus Mellié .................................... 6-22, 102-112

Cis hispidus (Paykull) ............................................ 6-22

Cis jacquemartii Mellié ............................ 6-22, 107-108

Cis lineatocribratus Mellié.............................. 6-22, 107

Cis micans (Fabricius)............................................. 6-22

Cis nitidus auct. nec (Fabricius) .............................. 6-22

Cis punctulatus Gyllenhal ...................................... 6-22

Cis quadridens Mellié ....................................... 102-112

Cis rugulosus Mellié ............................................... 6-22

Cixius similis (Kirschbaum) ................................. 72-90

Cladina ..................................................... 174, 177

Cladonia ................................................... 151, 153, 161

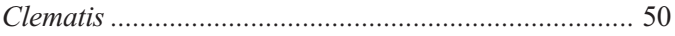

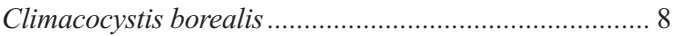

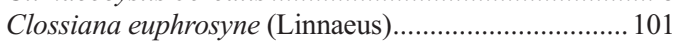

Clossiana selene (Denis \& Schiffermüller)................... 101

Clubiona caerulescens Koch ............................ 152, 188

Clubiona comta Koch ........................................ 72-90

Clubiona kulczynskii Lessert .............................. 152, 188

Clubiona lutescens Westring ............................... 152, 188

Clubiona neglecta P-Cambridge......................... 152, 188

Clubiona riparia Koch ...................................... 115-116

Clubiona stagnatilis Kulczynski..................113, 115, 117

Clubiona subsultans Thorell.............72-90, 152, 185, 188

Clubiona subtilis Koch....................................... 152, 188

Clubiona trivialis Koch......................... 72-90, 152, 188

Clytus arietis (Linnaeus) ..................................... 57-59

Cnephalocotes obscurus (Blackwall)..................159, 189

Cnephia dacotensis (Dryar \& Shannon) ...................... 127

Cnephia eremites Shewell .................................. 124-127

Cnephia pallipes (Fries) ..................................... 124-127

Coccotorus chaoi Chen .................................... 142-148

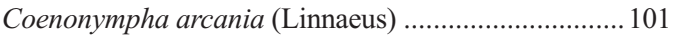

Coenonympha pamphilus (Linnaeus) ............................ 101

Coenosia lacteipennis (Zetterstedt) ........................ 72-90

Coenosia means Meigen ...................................... 72-90

Coenosia mollicula (Fallén) .................................. 72-90

Coenosia octopunctata (Zetterstedt) ....................... 72-90

Colastes Haliday ..................................................... 225

Colobostema ..................................................... 72-90

Conioscinella sordidella (Zetterstedt)......................72-90

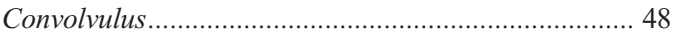

Coptoformica Müller .................................................. 217

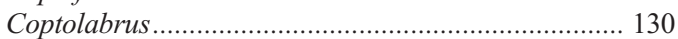


Cordyla murina Winnertz................................... 72-90

Coriachne depressa (Koch)................................... 72-90

Cornicularia cuspidata ........................................... 171

Cornicularia karpinskii .................................... 171-172

Cornicularia unicornis......................................... 172

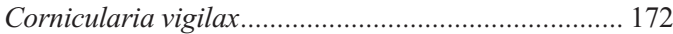

Corticaria alleni Johnson......................................... 6-22

Corticaria elongata (Gyllenhal) ............................... 6-22

Corticaria impressa (Olivier) ................................... 6-22

Corticaria lapponica (Zetterstedt)........................... 6-22

Corticaria longicollis (Zetterstedt) .......................... 6-22

Corticaria rubripes Mannerheim .............................. 6-22

Corticaria serrata (Paykull)................................... 6-22

Corticarina lambiana Sharp................................ 72-90

Corticarina latipennis (Sahlberg) ........................ 72-90

Corticeus linearis (Fabricius) ................................. 72-90

Cortinicara gibbosa (Herbst) ................................... 6-22

Corynoptera boletiphaga (Lengersdorf) ...................72-90

Corynoptera minima (Meigen)................................72-90

Corynoptera trepida (Winnertz) ............................. 72-90

Corynoptera Winnertz ............................................... 107

Cosmiotes exatella (Herrich-Schäffer) .................... 72-90

Cratyna uliginosa (Lengersdorf) ............................ 72-90

Creoleon plumbeus (Oliver) ...................................... 222

Crustulina guttata (Wider) .................................179, 191

Cryphalus abietis (Ratzeburg)..................57-59, 198-210

Cryphalus saltuarius Weise ................................ 198-210

Cryphoeca silvicola (Koch)..............72-90, 155-156, 189

Cryptophagus abietis (Paykull)..................... 6-22, 72-90

Cryptophagus acutangulus Gyllenhal....................... 6-22

Cryptophagus angustus Ganglbauer....................... 72-90

Cryptophagus badius Sturm ..................................... 6-22

Cryptophagus dentatus (Herbst)............................. 6-22

Cryptophagus dorsalis Sahlberg............................ 72-90

Cryptophagus pallidus Sturm ................................... 6-22

Cryptophagus pseudodentatus Bruce ........................ 6-22

Cryptophagus pubescens Sturm ............................. 6-22

Cryptophagus saginatus Sturm ............................ 6-22

Cryptophagus scanicus (Linnaeus).......................... 6-22

Cryptophagus setulosus Sturm ................................. 6-22

Cryptopleurum minutum (Fabricius) ......................... 6-22

Crypturgus cinereus (Herbst).............................. 198-210

Crypturgus hispidulus Thomson ..............57-59, 198-210

Crypturgus pusillus (Gyllenhal) ...............57-59, 198-210

Crypturgus subcribrosus Eggers......................... 198-210

Ctenosciara hyalipennis (Meigen).......................... 72-90

Cueta lineosa (Rambur) .................................... 221, 223

Culicoides alatavicus Smatov \& Isimbekov ............72-90

Culicoides albicans (Winnertz) ...............................72-90

Culicoides chiopterus (Meigen) .............................. 72-90

Culicoides clintoni Boorman .................................. 72-90

Culicoides comosioculatus .................................. 72-90

Culicoides dewulfi Goetghebuer........................... 72-90

Culicoides grisescens Edwards ..............................72-90

Culicoides heliophilus Edwards ............................ 72-90

Culicoides impunctatus Goetghebuer ......................72-90

Culicoides kibunensis Tokunaga............................ 72-90

Culicoides obsoletus (Meigen) ............................... 72-90

Culicoides scoticus Downes \& Kettle...................... 72-90

Culicoides sphagnumensis Williams.......................... 72-90
Culicoides vexans (Staeger).................................... 72-90

Cuneopalpus cyanops (Rostock) ............................ 72-90

Cupido minimus (Fuessly).......................................... 101

Curtimorda bisignata (Redtenbacher) ...................... 6-22

Curtimorda maculosa (Naezen) ............................... 6-22

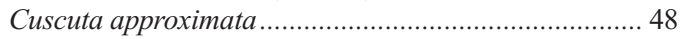

Cuscuta campestris .............................................. 48-51

Cuscuta epithymum............................................... 48-51

Cuscuta europaea ................................................ 48-51

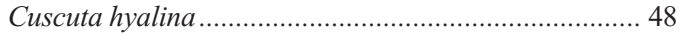

Cuscuta lupuliformis ........................................... 48-51

Cuscuta planiflora .................................................... 48

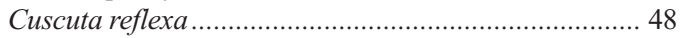

Cyaniris semiargus (Rottemburg)............................ 101

Cyclosa conica (Pallas) ...................72-90, 151, 185, 188

Cyllodes ater (Herbst) ............................................. 6-22

Cymberemaeus cymba (Nicolet)............................. 72-90

Cyphea curtula (Erichson) ..................................... 6-22

Cyphon ochraceus Stephens.................................. 72-90

Cyphon pubescens (Fabricius)................................ 6-22

Dacne bipustulata (Thunberg).................................. 6-22

Dactylopisthes video (Chamberlin \& Ivie)....................115

Daedalea quercina............................................... 8

Daedalopsis confragosa ........................................ 7-8

Daedalopsis tricolor ............................................... 7-8

Dalopius marginatus (Linnaeus) ............................72-90

Dasyhelea bensoni Edwards................................. 72-90

Dasyhelea biunguis Kieffer ..................................... 72-90

Dasyhelea europaea Remm ................................ 72-90

Dasyhelea ledi Remm ......................................... 72-90

Dasyhelea luteiventris Goetghebuer .......................... 72-90

Dasyhelea modesta (Winnertz)............................... 72-90

Dasyhelea norvegica Szadziewski \& Hagan............72-90

Dasyhelea parallela Remm................................. 72-90

Dasytes aerosus Kiesenwetter ...............................57-59

Dasytes cyaneus Fabricius...................................... 57-59

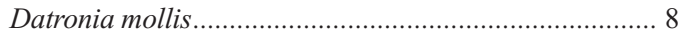

Dendroctonus micans (Kugelanni) ...................... 198-210

Dendryphantes rudis (Sundevall) .......................... 72-90

Denticollis linearis (Linnaeus) ................................. 6-22

Deuterosminthurus bicinctus (Koch) ....................... 72-90

Deuterosminthurus flavus (Gisin) .......................... 72-90

Diaperis boleti (Linnaeus)..................................... 6-22

Diapterobates humeralis (Hermann) ......................72-90

Dichocrysa flavifrons (Brauer) ...........................220-221

Dichocrysa prasina (Burmeister) ................................ 221

Dichocrysa venosa (Rambur)..................................... 220

Dicranomyia (Dicranomyia) autumnalis (Staeger) ........47

Dicranomyia (Dicranomyia) didyma (Meigen) .............4 47

Dicranomyia (Dicranomyia) handlirschi Lackschewitz 47

Dicranomyia (Dicranomyia) humidicola Osten-Sacken 44

Dicranomyia (Dicranomyia) modesta (Meigen)............47

Dicranomyia (Idiopyga) stigmatica (Meigen) ...............47

Dicranomyia s.str. mitis (Meigen) ......................... 72-90

Dictyna arundinacea (Linnaeus) ..............72-90, 115-117,

153,188

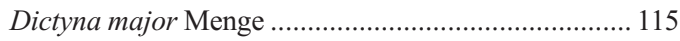

Dictyna pusilla Thorell ..............................153, 185, 188

Dicymbium tibiale (Blackwall) ...........................159, 189

Dicyrtomina minuta (Fabricius) ............................. 72-90 
Dielocroce ephemera (Gerstäcker) ............................ 223

Dienerella filum (Aubé) ............................................ 6-22

Dinaraea aequata (Erichson) .................................... 6-22

Dinaraea angustula (Gyllenhal).............................. 6-22

Dinaraea linearis (Gravenhorst) ............................. 6-22

Diosphilus dispar (Nees).......................................... 106

Diplocentria bidentata (Emerton)......................159, 189

Diplocentria rectangulata (Emerton)..................159, 189

Diplocephalus latifrons (P-Cambridge)........159, 183, 189

Diplocephalus picinus (Blackwall) ..............159-160, 189

Diplostyla concolor (Wider) .............................. 160, 189

Dipoena torva (Thorell) ....................72-90, 179-180, 191

Discobola annulata (Linnaeus) ..........................47, 107

Discobola caesarea (Osten-Sacken) ...................47, 107

Dismodicus bifrons (Blackwall).........................160, 189

Dismodicus elevatus (Koch) ................... 72-90, 160, 189

Distoleon curdicus Hölzel ................................... 222-223

Distoleon tetragrammicus (Fabricius) .................222-223

Dolichopus annulipes Zetterstedt ......................... 72-90

Dolichopus discifer Stannius ................................. 72-90

Dolomedes fimbriatus (Clerck)...........................177, 191

Dolopsidea Hincks...................................................... 225

Dorcatoma chrysomelina Sturm .............................. 6-22

Dorcatoma dresdensis Herbst.................................. 6-22

Dorcatoma flavicornis (Fabricius) ........................... 6-22

Dorcatoma lomnickii Reitter ................................... 6-22

Dorcatoma punctulata Mulsant \& Rey ......6-22, 106-108

Dorcatoma robusta Strand ....................................... 6-22

Dorcatoma substriata Hummel ................................ 6-22

Doryctomorpha Ashmead .......................................225

Dorypteryx domestica (Smithers) ........................... 72-90

Drapetisca socialis (Sundevall) .......................... 160, 189

Drassodes cupreus (Blackwall) .................................. 115

Drassodes mirus Platnick \& Shadab............................ 116

Drassodes pubescens (Thorell)...........................153, 188

Drassyllus praeficus (Koch) .............................. 153, 188

Drassyllus pusillus (Koch).........................153, 184, 188

Dromius agilis Fabricius .................................... 72-90

Dromius angustus Brullé...................................... 72-90

Dromius fenestratus (Fabricius) ............................ 72-90

Dromius schneideri Crotch...................................... 72-90

Dromius spilotus (Illiger)...........................57-59, 72-90

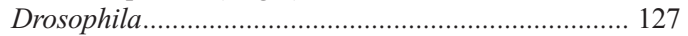

Dryocoetes alni (Georg) ................................... 198-210

Dryocoetes autographus (Ratzeburg) ................. 198-210

Dryocoetes baikalicus Reitter............................. 198-210

Dryocoetes hectographus Reitter........................ 198-210

Eboria fausta ................................................. 167-168

Ectobius lapponicus (Linnaeus) ............................72-90

Ectodemia minimella (Zetterstedt)..........................72-90

Ectrepesthoneura pubescens (Zetterstedt) ...............72-90

Elatophilus nigricornis (Zetterstedt)......................72-90

Eledona agaricola (Herbst).................................. 6-22

Elephantomyia krivosheinae Savchenko.................45, 47

Elipsocus hyalinus (Stephens) ............................... 72-90

Emblyna annulipes (Blackwall).................................. 115

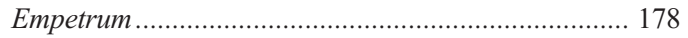

Empis (Anacrostichus) lucida Zetterstedt.................72-90

Enderleinella obsoleta (Stephens) ..........................72-90

Enicmus fungicola Thomson ......................... 6-22, 72-90
Enicmus rugosus (Herbst)

6-22

Ennearthron cornutum (Gyllenhal) ..............6-22, 102-112

Ennearthron laricinum (Mellié) .................6-22, 106, 108

Ennearthron palmi Lohse....................................... 6-22

Enoplognatha serratosignata (Koch) ......................... 115

Entelecara congenera (Cambridge)........................72-90

Entelecara congenera (P-Cambridge) .................160, 189

Entelecara erythropus (Westring) ....................... 160, 189

Entelecara flavipes (Blackwall).............................. 72-90

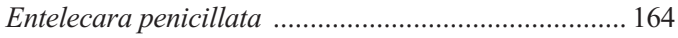

Entomobrya albocincta (Templeton) ......................72-90

Entomobrya lanuginosa (Nicolet) ......................... 72-90

Entomobrya marginata (Tullberg) ......................... 72-90

Entomobrya nivalis (Linnaeus) ............................. 72-90

Epidapus gracilis (Walker).................................. 72-90

Epilobium .............................................................. 3

Episernus angusticollis Thomson.............................. 72-90

Episinus angulatus (Blackwall)...............72-90, 180, 191

Epuraea biguttata (Thunberg).................................. 6-22

Epuraea concurrens Sjöberg .................................. 6-22

Epuraea contractula Sahlberg................................. 6-22

Epuraea distincta (Grimmer) .................................. 6-22

Epuraea limbata (Fabricius).................................... 6-22

Epuraea pygmaea (Gyllenhal) ............................. 72-90

Epuraea silacea (Herbst) ........................................ 6-22

Epuraea unicolor (Olivier)..................................... 6-22

Epuraea variegata (Herbst)..................................... 6-22

Eremocoris abietis (Linnaeus)................................ 72-90

Erigone atra Blackwall......................160-161, 185, 189

Erigone dentipalpis (Wider).......................161, 185, 189

Erigone psychrophila Thorell...................................... 116

Erigonella hiemalis (Blackwall) ..................161, 184, 189

Erioptera (Erioptera) sordida Zetterstedt..................... 47

Eristalis alpina (Panzer)........................................... 122

Eristalis arbustorum (Linnaeus)............................... 122

Eristalis interrupta (Poda) ..........................119-120, 122

Eristalis intricaria (Linnaeus) .................................. 122

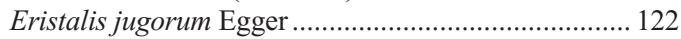

Eristalis lineata (Harris)........................................... 122

Eristalis nigritarsis Macquart.................................... 122

Eristalis pertinax (Scopoli) .......................................... 122

Eristalis picea (Fallén) ............................................... 122

Eristalis rupium Fabricius ................................ 119, 122

Eristalis similis (Fallén) .............................................. 122

Eristalis tecta sp. n. ......................................... 119-123

Eristalis tenax (Linnaeus) ......................................... 122

Ernobius nigrinus (Sturm)..................................... 72-90

Ernoporicus caucasicus (Lindemann) ................. 198-210

Ernoporus tiliae (Panzer) .................................. 198-210

Ero furcata (Villers)................................... 177, 184, 191

Eubeckerella Narendran ............................................ 142

Eueremaeus oblongus (Koch) .............................. 72-90

Euophrus erratica............................................... 178

Euophrys frontalis (Walckenaer).................177-178, 191

Euophrys maculata (Wider) .............................. 177-178

Euophrys proszynskii Logunov, Cutler \& Marusik

115,117

Eupelops acromios (Hermann) ............................. 72-90

Euphorbia cyparissias .............................................. 139

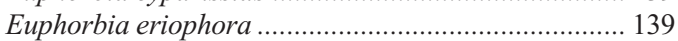


Euphorbia esula.....

139

Euphorbia falcata

$139-140$

Euphorbia lucida .................................................... 139

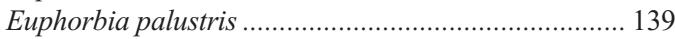

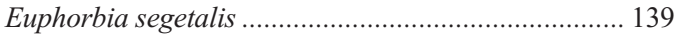

Euphydryas aurinia (Rottemburg) .....................231-241

Euphylidorea phaeostigma (Schummel) ...........47, 72-90

Euplectus decipiens Raffray ................................. 72-90

Euryopis flavomaculata (Koch)..........................180, 192

Euryusa castanoptera Kraatz ................................. 6-22

Eusimulium cf. cryophilum Rubtsov......................72-90

Euthyneura myrtilli Macquart ............................... 72-90

Evarcha falcata (Clerck) .................72-90, 178, 184, 191

Evarcha proszynskii Marusik \& Logunov......113, 115, 117

Exochomus quadripustulatus (Linnaeus) ..................72-90

Exotelia dodecella (Linnaeus) ................................. 72-90

Fabriciana adippe (Denis \& Schiffermüller) ..................101

Fabriciana niobe (Linnaeus)........................................ 101

Fagus ................................................................. 120

Fannia canicularis (Linnaeus) .............................. 72-90

Fannia carbonaria (Meigen)................................. 72-90

Fannia umbrosa (Stein)....................................... 72-90

Fannia verrallii (Stein) ......................................... 72-90

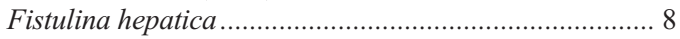

Fomes fomentarius.............................................. 6-22

Fomitopsis pinicola ..................................... 9, 102-112

Fomitopsis rosea ..................................................... 110

Forcipomyia acidicola (Tokunaga)..........................72-90

Forcipomyia albostyla Remm ..............................72-90

Forcipomyia bipunctata (Linnaeus)........................72-90

Forcipomyia brevipennis (Macquart)...................... 72-90

Forcipomyia ciliata (Winnertz) ............................. 72-90

Forcipomyia fuliginosa (Meigen) .......................... 72-90

Forcipomyia hygrophila Kieffer ........................... 72-90

Forcipomyia kaltenbachi (Winnertz) .......................72-90

Forcipomyia monilicornis (Coquillett) ................... 72-90

Forcipomyia nigra (Winnertz).............................. 72-90

Forcipomyia nigrans Remm.............................. 72-90

Forcipomyia palustris (Meigen) .............................72-90

Forcipomyia titillans (Winnertz) ........................... 72-90

Formica (Serviformica) fusca Linnaeus....................... 211

Formica aquilonia Yarrow....................... 72-90, 213-217

Formica exsecta Nylander ........................................ 216

Formica fusca Linnaeus ......................................... 72-90

Formica lemani Bondroit ...................................... 72-90

Formica Linnaeus .............................................. 166, 211

Formica lugubris Zetterstedt................... 72-90, 213-217

Formica rufa Linnaeus ............................................ 170

Formica truncorum Fabricius.............................213-217

Fraxinus rhynchophylla .................................. 130

Funalia trogii.......................................................... 9

Fuscozetes fuscipes (Koch) ................................... 72-90

Ganoderma applanatum ............................................. 9

Ganoderma lipsiense ................................................... 9

Gastrodes grossipes (DeGeer) .............................. 72-90

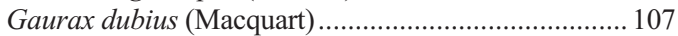

Gaurax maculipennis (Zetterstedt) ............................ 107

Geotrupes stercorosus (Scriba)................................ 6-22

Gibbaranea omoeda (Thorell) .............151-152, 185, 188

Glischrochilus grandis (Reitter) .............................. 6-22
Glischrochilus grandis (Tournier) ........................... 6-22

Glischrochilus hortensis (Geoffroy) .......................... 6-22

Globicornis emarginata (Gyllenhall)........................ 6-22

Gloeophyllum abietinum ............................................. 9

Gloeophyllum protractum ........................................... 9

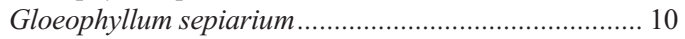

Gloeophyllum trabeum .............................................. 10

Glycyrrhiza glabra................................................... 3

Gnaphosa bicolor (Hahn)..................................154, 188

Gnaphosa borea Kulczynski ..................................... 116

Gnaphosa microps Holm ............................ 116, 154, 188

Gnaphosa montana (Koch) ............................... 154, 188

Gnaphosa nigerrima Koch ............................... 115-116

Gnaphosa similis Kulczynski ..................................... 115

Gnaphosa sticta Kulczynski..................................... 116

Gnathoncus buyssoni Auzat ...................................... 6-22

Gnathotrichus materiarius (Fitch) ....................... 198-210

Gnophomyia acheron Alexander .......................... 45, 47

Gonatium rubellum (Blackwall) .........................161, 189

Gongylidiellum latebricola (P-Cambridge)..........161, 189

Gongylidiellum murcidum Simon ........................161, 185

Gongylidium rufipes (Linnaeus) .........................161, 189

Graphopsocus cruciatus (Linnaeus) .......................72-90

Graptodera longicollis Allard .................................... 139

Grynocharis oblonga (Linnaeus).............................. 6-22

Gyrophaena affinis Mannerheim .............................. 6-22

Gyrophaena bihamata Thomson ............................. 6-22

Gyrophaena boleti (Linnaeus) ................................. 6-22

Gyrophaena fasciata (Marsham) ............................. 6-22

Gyrophaena gentilis Erichson .................................. 6-22

Gyrophaena joyi Wendeler........................................ 6-22

Gyrophaena joyioides Wusthoff.............................. 6-22

Gyrophaena lucidula Erichson ................................ 6-22

Gyrophaena manca Erichson ................................. 6-22

Gyrophaena minima Erichson ................................ 6-22

Gyrophaena nana (Paykull) ................................... 6-22

Gyrophaena obsoleta Ganglbauer ........................... 6-22

Gyrophaena orientalis Strand.................................... 6-22

Gyrophaena poweri Crotch .................................. 6-22

Gyrophaena pulchella Heer .................................... 6-22

Gyrophaena strictula Erichson.................................. 6-22

Gyrophaena transversalis Strand ............................. 6-22

Hahnia mengei Chyzer \& Kulczynski ........................ 156

Hahnia nava (Blackwall) ..................................156, 189

Hahnia ononidum Simon................................... 156, 189

Hahnia pusilla Koch ...................................... 156, 189

Hallomenus axillaris (Illiger) ................................ 6-22

Hallomenus binotatus (Quensel) ............................ 6-22

Haltica atrocaerulea Stephens ................................... 139

Haltica violacea Koch ............................................. 139

Hapalopilus rutilans ................................................... 10

Haplodrassus cognatus (Westring) .....................154, 188

Haplodrassus hiemalis (Emerton) ............................. 116

Haplodrassus moderatus (Kulczynski)...............154, 188

Haplodrassus signifer (Koch)............................ 154, 188

Haplodrassus silvestris (Blackwall)....................154, 188

Haplodrassus soerenseni (Strand)...............154-155, 188

Haploglossa villosula (Stephens) ............................ 6-22

Haplothrips aculeatus (Fabricius) .......................... 72-90

Helina daicles (Walker)....................................... 72-90 
Helina evecta (Harris)

$72-90$

Helina subvittata (Séguy)

72-90

Heliophanus camtschadalicus Kulczynski.

Heliophanus dampfi Schenkel

Heliophanus dubius Koch ....

115

Heliophanus patagiatus Thorell

Helophora insignis (Blackwall)

78, 191

178,191

$162,184,189$

Hemileius initialis (Berlese) .................................. 72-90

Hesperia comma (Linnaeus) ....................................... 101

Heterobasidion ..................................................... 10

Heterobasidion annosum ................................................. 7

Heterobasidion parviporum ......................................... 7

Heterosminthurus claviger Gisin ........................... 72-90

Heterothera firmata (Hübner) ................................ 72-90

Hilaira hermiosa (Thorell) ............................... 162, 189

Hilara intermedia (Fallén) ..................................... 72-90

Hilara interstincta (Fallén)..................................... 72-90

Homalota plana (Gyllenhal) .................................... 6-22

Hoplandrothrips williamsianus Priesner..................72-90

Hoplothrips ulmi (Fabricius) ................................. 72-90

Hybos grossipes (Linnaeus).................................... 72-90

Hydriomena furcata (Thunberg) ............................. 72-90

Hydrophoria lancifer (Harris) ............................... 72-90

Hylaea fasciaria (Linnaeus) ................................ 72-90

Hylastes angustatus (Herbst) .............................. 198-210

Hylastes attenuatus Erichson.............................. 198-210

Hylastes brunneus Erichson ............................... 198-210

Hylastes cunicularius Erichson .......................... 198-210

Hylastes fraxini (Panzer) ................................... 198-210

Hylastes opacus Erichson................................... 198-210

Hylastes plumbeus Blandford ............................. 198-210

Hylastes varius (Fabricius) ................................... 198-210

Hyledelphax elegantula (Boheman) ....................... 72-90

Hylemya partita (Meigen) ...................................... 72-90

Hylesinus crenatus (Fabricius)............................ 198-210

Hylobius abietis (Linnaeus).................................... 72-90

Hylobius piceus (DeGeer) .................................... 72-90

Hylocomium splendens ........................................... 150

Hylurgops glabratus (Zetterstedt).......................198-210

Hylurgops palliatus (Gyllenhal)...............72-90, 198-210

Hylurgus ligniperda (Fabricius).......................... 198-210

Hyoscymus niger................................................. 140

Hyphodontia paradoxa .......................................... 10

Hypochthonius rufulus Koch.................................. 72-90

Hypogastrura socialis (Uzel) ................................ 72-90

Hypsosinga sanguinea (Koch)...........................152, 188

Incestophantes kochiellus (Strand)......................162, 189

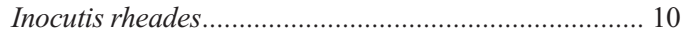

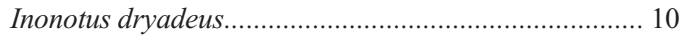

Inonotus hispidus ............................................... 10

Inonotus obliquus ..................................................... 10

Inonotus radiatus ................................................ 10-11

Inonotus rheades........................................................... 10

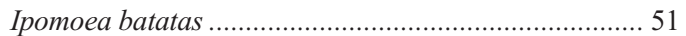

Ips acuminatus (Gyllenhal) ................................ 198-210

Ips amitinus (Eichhoff)...................................... 198-210

Ips cembrae (Heer)............................................ 198-210

Ips duplicatus Sahlberg.......................... 72-90, 198-210

Ips sexdentatus (Börner)..................................... 198-210

Ips subelongatus Motschulsky............................ 198-210
Ips typographus (Linnaeus) ............................ 198-210

Isotoma anglicana Lubbock .................................... 72-90

Isotoma hiemalis Schött ........................................ 72-90

Isotoma notabilis Schäffer................................... 72-90

Isotoma olivacea Tullberg .................................. 72-90

Isotoma viridis Bourlet........................................ 72-90

Italochrysa italica (Rossi) ................................... 220, 223

Iteaphila nitidula (Zetterstedt) .............................. 72-90

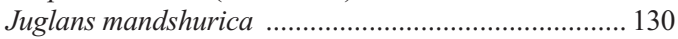

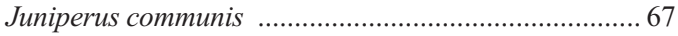

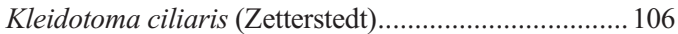

Korynetes caeruleus (DeGeer) ................................. 6-22

Laetiphorus sulphureus ....................................... 6-22

Larinioides cornutus (Clerck)..................................... 115

Larinioides patagiatus (Clerck)................................... 115

Larix ............................................................ 129, 207

Lasaeola tristis (Hahn) ....................................... 180, 192

Lasiomma atricauda (Zetterstedt) ......................... 72-90

Lasiomma cuneicorne (Zetterstedt) .........................72-90

Lasiosoma nitens Winnertz ......................................... 194

Lasius (Cautolasius) flavus Fabricius .................212-217

Lasius Fabricius ............................................... 212-218

Lasius fuliginosus (Latreille) ................................ 72-90

Lasius niger (Linnaeus) ................................... 212-217

Lasius platythorax Seifert...................................212-217

Lasius psammophilus Seifert...................................... 216

Latithorax faustus ........................................... 167-168

Latridius brevicollis (Thomson) ............................... 6-22

Latridius consimilis Mannerheim ............................. 6-22

Latridius hirtus Gyllenhal ..................................... 6-22

Latridius minutus (Linnaeus) ................................... 6-22

Ledum .................................................. 151, 156-183

Leiestes seminigra (Gyllenhall).............................. 6-22

Leiopus nebulosus (Linnaeus) ...............................57-59

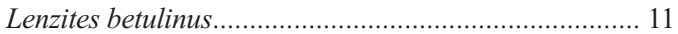

Lepidium ...................................................... 139-140

Lepidocyrtus lignorum (Fabricius) ........................ 72-90

Lepinotus patruelis Pearman ................................... 72-90

Lepthyphantes alacris ............................................. 169

Lepthyphantes mengei............................................ 169

Lepthyphantes obscurus (Blackwall) ....................... 72-90

Lepthyphantes pallidus (Cambridge) .......................72-90

Lepthyphantes tenebricola................................. 169-170

Leptophlebia vespertina (Linnaeus) ........................72-90

Leptothorax acervorum (Fabricius) ....................213-217

Leptothorax muscorum (Nylander).....................213-217

Leptusa fumida (Erichson) ............................................. 110

Leptusa pulchella (Mannerheim)....6-22, 72-90, 108, 110

Leptusa ruficollis (Erichson).......................57-59, 72-90

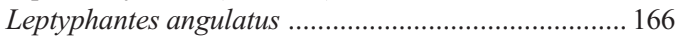

Leptyphantes angulipalpis ......................................... 157

Leptyphantes antroniensis Schenkel ...................162, 189

Leptyphantes cochiellus .......................................... 160

Leptyphantes exiguus Holm .......................................... 160

Leptyphantes expunctus ............................................. 156

Leptyphantes obscurus............................................... 165

Leptyphantes pallidus ................................................. 166

Lertha extensa (Oliver) .............................................. 222

Lertha sheppardi (Kirby) ............................................ 223

Lestodiplosis coni (Kieffer) .................................... 72-90 
Lestodiplosis polypori (Loew).

Lestremia cinera Macquart.

$72-90$

Lestremia leucophaea (Meigen).....

72-90

Leucophenga Mik

107

Leucophenga quinquemaculata Strobl ........................ 107

Leucorrhinia dubia (Van der Linden) ......................72-90

Leuctra nigra (Olivier)......................................... 72-90

Liacarus subterraneus (Koch).............................. 72-90

Liebstadia similis (Michael) .................................. 72-90

Limnephilus centralis Curtis................................. 72-90

Limnephilus vittatus (Fabricius) ........................... 72-90

Limnobia indigenoides Alexander .........................42, 44

Limnophora nigripes (Robineau-Desviody)............72-90

Limnozetes rugosus Sellnick ................................. 72-90

Limonia (Limonia) badia Alexander ............................. 42

Limonia (Limonia) indigenoides Alexander .................. 44

Limonia alpicola (Lackschewitz) ................................... 44

Limonia badia (Walker) ........................................ 41-47

Limonia indigena (Osten Sacken)...........................44-45

Limonia indigena jacksoni (Alexander)........................ 45

Limonia indigena loloensis Alexander ......................... 45

Limonia macrostigma (Schummel).........................44-45

Limonia sylvicola (Schummel).................................. 47

Limonius aeneoniger (DeGeer) ............................. 72-90

Limothrips denticornis Haliday .............................. 72-90

Linyphia clathrata ........................................... 164-165

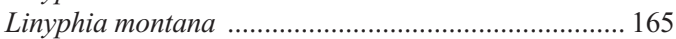

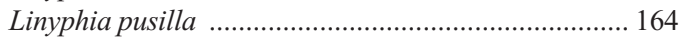

Liposcelis silvarum (Kolbe) ................................. 72-90

Lipsothrix ecucullata Edwards ............................ 45, 47

Lissonota Gravenhorst .......................................... 106

Litargus connexus (Geoffroy)................................... 6-22

Lithobius borealis Meinert ..................................... 72-90

Lochmaea suturalis (Thomson)............................. 72-90

Loensia fasciata (Fabricius) ................................... 72-90

Lonchaea laxa Collin ......................................... 72-90

Lonchaea stackelbergi Czerny ............................... 72-90

Longitarsus jacobaea (Waterhouse) ............................ 140

Lophomma punctatum (Blackwall).....................162, 189

Lordithon exoletus (Erichson) ................................... 6-22

Lordithon lunulatus (Linnaeus) .................................. 6-22

Lordithon thoracicus (Fabricius) .............................. 6-22

Loricula pselaphiformis Curtis ............................... 72-90

Lycaena hippothoe Linnaeus ........................................ 101

Lycaena phlaeas (Linnaeus)........................................ 101

Lycaena virgaureae (Linnaeus) .................................. 101

Lycophotia porphyrea (Denis \& Schiffermüller)......72-90

Lycoriella globiceps (Becher).................................72-90

Lycoriella latilobata (Menzel \& Mohrig) ................72-90

Lycoriella solani (Winnertz) ................................. 107-108

Lycosa chleata (Müller) ............................................... 174

Lycosa hyperborea ................................................... 174

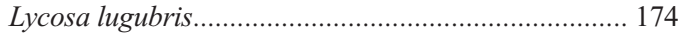

Lycosa paludicola ....................................................... 174

Lycosa palustris ……..................................... 174-175

Lycosa pernix Thorell ................................................ 175

Lycosa pullata............................................................ 175

Lycosa riparia ........................................................ 175

Lycosa saccata (Linnaeus) ............................................ 174

Lycosa tarsalis Thorell ..................................... 174-175
Lygus punctatus (Zetterstedt) ............................. 72-90

Lymantor coryli (Perris) ....................................... 198-210

Lypha dubia (Fallén) ............................................. 72-90

Lysitermoides van Achterberg .................................... 225

Macrargus boreus Holm .................................162, 189

Macrargus carpenteri (P-Cambridge).................162, 189

Macrargus multesimus (P-Cambridge) ................162, 189

Macrargus rufus (Wider) ........................... 162-163, 189

Magdalis violacea (Linnaeus) ................................57-59

Malthodes brevicollis Paykull .............................. 72-90

Malthodes fuscus Waltl ........................................ 72-90

Malthodes guttifer Kiesenwetter............................. 72-90

Malthodes pumilus Brebisson................................ 72-90

Maniola jurtina (Linnaeus) ......................................... 101

Mantispa scabricollis McLachlan ..............................223

Maro lehtineni Saaristo .....................................163, 189

Maro lepidus Casemir ........................................ 163, 189

Maro sublestus Falconer.................................... 163, 189

Maso sundevalli (Westring)..............72-90, 163, 183, 189

Mastigusa arietina (Thorell)..............................153, 188

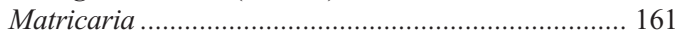

Mecynargus tungusicus (Eskov) ................................... 116

Medetera abstrusa Thuneberg..................................... 107

Medetera impigra Collin........................................... 107

Medetera setiventris Thunberg ............................... 72-90

Medetera striata Parent .......................................... 72-90

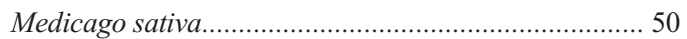

Medina collaris (Fallén)........................................ 72-90

Megarthrus denticollis (Beck) ............................... 6-22

Megarthrus depressus (Paykull) ............................... 6-22

Megarthrus hemipterus (Illiger) .............................. 6-22

Megarthrus nitidulus Kraatz................................... 6-22

Megarthrus sinuatocollis Lacordaire ............ 6-22, 72-90

Megaselia aculeata (Schmitz) .............................. 72-90

Megaselia aquilonia Schmitz ................................ 72-90

Megaselia armata (Wood) .......................................... 107

Megaselia cothurnata (Schmitz) ............................ 72-90

Megaselia eccoptomera Schmitz ............................ 72-90

Megaselia emarginata (Wood) .............................. 72-90

Megaselia fusciclava Schmitz .............................. 72-90

Megaselia gartensis Disney .................................... 72-90

Megaselia giraudii .............................................. 72-90

Megaselia nigriceps (Loew).................................. 72-90

Megaselia pleuralis (Wood) ................................... 72-90

Megaselia pusilla (Meigen).................................... 72-90

Megaselia robusta Schmitz ................................... 72-90

Megaselia rufipes (Meigen) ....................................... 107

Megaselia spinigera (Wood) ................................. 72-90

Megasternum obscurum (Marsham)........................6-22

Meioneta affinis (Kulczynski) ...........................163, 190

Meioneta beata P-Cambridge..................................... 163

Meioneta gulosa (Koch) ...................................163, 190

Meioneta rurestris (Koch) .............................. 163, 190

Melanagromyza albocilia Hendel................................. 48

Melanagromyza cuscutae Hering ...........................48-52

Melandrya dubia (Schaller)...................................... 6-22

Melitaea cinxia (Linnaeus) ................................. 101, 239

Mellicta athalia (Rottemburg).................................... 101

Mellicta britomartis (Assmann) ................................... 101

Menozziola obscuripes (Schmitz) ........................... 72-90 


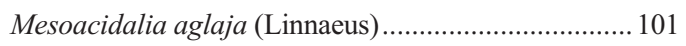

Mesopsocus unipunctatus (Müller)..........................72-90

Meta mengei ........................................................ 179

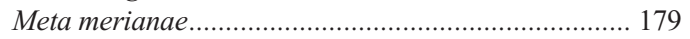

Metalimnobia quadrimaculata (Linnaeus) ....................4 47

Metalimnobia zetterstedti (Tjeder) ............................... 47

Metaspathius Brues.................................................... 225

Metellina mengei (Blackwall)............................ 179, 191

Metellina merianae (Scopoli) ............................. 179, 191

Metopia campestris (Fallén)................................. 72-90

Micaria aenea Thorell.........................116-117, 155, 188

Micaria alpina Koch............................................... 116

Micaria lenzi Bösenberg ........................................... 116

Micaria pulicaria (Sundevall) ......................... 155, 188

Micaria rossica Thorell ...................................... 116-117

Micaria silesiaca Koch ................................... 155, 188

Micrargus apertus ..................................... 163-164, 190

Micrargus herbigradus (Blackwall)....................164, 190

Micreremus brevipes (Michael) .............................. 72-90

Microcentria pusilla Schenkel..................................... 159

Microcentria rectangulata ........................................ 159

Microlinyphia pusilla (Sundevall) ................116, 164, 190

Micrommata roseum (Clerck) ................................... 179

Micrommata virescens (Clerck) ........................179, 191

Micrommata viridissima (DeGeer)............................. 179

Microneta viaria (Blackwall) .............................164, 190

Micropterix aureatella (Scopoli) ............................ 72-90

Minettia lupulina (Fabricius) .................................. 72-90

Minicia marginella (Wider).............................. 164, 190

Minilimosina $v$-atrum (Villeneuve).......................72-90

Minyriolus pusillus (Wider) .................... 72-90, 164, 190

Misumena vatia (Clerck) .......................................... 115

Moebelia penicillata (Westring)..............72-90, 164, 190

Molinia ...................................................... 151-183

Molophilus s.str. bifidus Goetghebuer......................72-90

Molorchus minor (Linnaeus) .................................. 72-90

Mycetophagus ater (Reitter)................................. 6-22

Mycetophagus decempunctatus Fabricius................. 6-22

Mycetophagus multipunctatus Fabricius................... 6-22

Mycetophagus piceus (Fabricius) ............................ 6-22

Mycetophagus quadriguttatus Müller....................... 6-22

Mycetophagus quadripustulatus (Linnaeus) ............. 6-22

Mycetophagus salicis Briosut de Barneville ..............6-22

Mydaea electa (Zetterstedt) ................................... 72-90

Mydaea sootryeni Ringdahl.................................. 72-90

Myrica ................................................... 151-183

Myrmecaelurus maior McLachlan.....................221, 223

Myrmecaelurus trigrammus (Pallas) ..........................221

Myrmedobia exilis (Fallén).................................... 72-90

Myrmeleon inconspicuus Rambur..........................221, 223

Myrmica lobicomis Nylander ................................ 72-90

Myrmica rubra (Linnaeus) ............................... 213-217

Myrmica ruginodis Nylander ............................... 72-90

Myrrha octodecimguttata (Linnaeus) ..................... 72-90

Myzia oblongoguttata (Linnaeus) ........................... 72-90

Nanhermannia coronata Berlese ............................72-90

Nedroleon striatus (Klug)................................. 222-223

Need abeer Linnaeus.............................................. 1-256

Nemapogon Schrank .................................................. 107

Nemoptera sinuata Oliver .............................................. 222
Nemoura cinerea (Retzius)................................... 72-90

Nemurella pictetii Klapalek.................................... 72-90

Neoleucopis obscura (Haliday) .............................. 72-90

Neoleucopis tapiae (Blanchard) .............................72-90

Neolimonia dumetorum (Meigen) ................................ 47

Neomida haemorrhoidalis (Fabricius) ......................6-22

Neon reticulatus (Blackwall)............72-90, 178, 184, 191

Neottiura bimaculata (Linnaeus) ........................ 180, 192

Neriene clathrata (Sundevall) ..............164-165, 184, 190

Neriene marginata (Koch) ....................................... 165

Neriene montana (Clerck) ............................... 165, 190

Neriene peltata (Wider) .............................. 165, 185, 190

Neriene radiata (Walckenaer)............................ 165, 190

Neuroleon assimilis Navás ................................ 221, 223

Neuroleon egenus (Navás) ......................................... 222

Neuroleon microstenus (McLachlan)...........................222

Neuroleon tenellus (Klug) ........................................... 221

Nicrophorus vespilloides Herbst............................... 6-22

Nossidium pilosellum (Marsham) ............................ 6-22

Obscuriphantes obscurus (Blackwall) ................ 165, 190

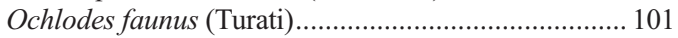

Ocnerostoma friesei Svensson................................ 72-90

Ocnerostoma piniarella Zeller............................... 72-90

Octotemnus glabriculus (Gyllenhal)........................ 6-22

Ocydromia glabricula (Fallén) ................................ 72-90

Odontocepheus elongatus (Michael) .......................72-90

Oedothorax apicatus (Blackwall) ....................... 165, 190

Oedothorax gibbosus (Blackwall)................165, 184, 190

Oedothorax gibbosus tuberosus (Blackwall) ............... 165

Oedothorax retusus (Westring) ....................165-166, 190

Oiceoptoma thoracica (Linnaeus) ............................ 6-22

Oligota granaria Erichson ...................................... 6-22

Omalium caesum Gravenhorst ............................ 72-90

Omalium rivulare (Paykull) .................................. 6-22

Oncophanes Foerster ......................................... 225-226

Oncophanes tobiasi Zaykov........................................ 225

Oncopsis flavicollis (Linnaeus) .............................. 72-90

Oppiella nova (Oudermans) ................................... 72-90

Orchesella bifasciata Nicolet ................................ 72-90

Orchesella cincta (Linnaeus)................................. 72-90

Orchesella flavescens (Bourlet) ................................ 72-90

Orchesia fasciata (Illiger) ...................................... 6-22

Orchesia fusiformis Solsky...................................... 6-22

Orchesia micans (Panzer) ........................................ 6-22

Oreoneta alpina (Eskov) .................................... 116-117

Oreonetides vaginatus (Thorell) ......................... 166, 190

Oribatula tibialis (Nicolet) ..................................... 72-90

Ormosia (Ormosia) ruficauda (Zetterstedt) ...................47

Ormosia ruficauda (Zetterstedt) ............................ 72-90

Ormyrulus Boucek .................................................... 142

Ormyrus coccotori sp. n. ................................... 142-148

Ormyrus flavotibialis Yasumatsu \& Kamijo ................. 147

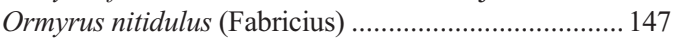

Orthoperus atomus (Gyllenhal)................................ 6-22

Orthoperus corticalis (Redtenbacher) .......................... 6-22

Orthoperus improvisus Bruce.................................. 6-22

Orthoperus punctulatus Reitter ............................... 6-22

Orthoperus rogeri Kraatz........................................ 6-22

Orthotomicus laricis (Fabricius) ......................... 198-210

Orthotomicus longicollis (Gyllenhal)................... 198-210 
Orthotomicus proximus (Eichhoff)

$198-210$

Orthotomicus starki Spessivtseff

$198-210$

Orthotomicus suturalis (Gyllenhal)..........57-59, 198-210

Oryphantes angulatus (P-Cambridge) .................166, 190

Oscinella frit Linnaeus ......................................... 72-90

Ostoma ferruginea (Linnaeus) ................................. 6-22

Oxypoda alternans (Gravenhorst) ............................ 6-22

Oxyporus rufus (Linnaeus)....................................... 6-22

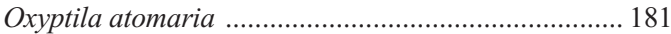

Oxyptila praticola ............................................ 181-182

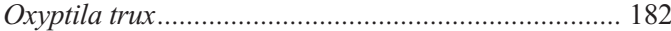

Oxythrips ajugae Uzel........................................ 72-90

Oxythrips bicolor (Reuter) ................................... 72-90

Ozyptila arctica Kulczynski ...................................... 117

Ozyptila atomaria (Panzer) .............................. 181, 192

Ozyptila praticola (Koch) ....................181-182, 184, 192

Ozyptila trux (Blackwall) ................................... 182, 192

Pachycerina seticornis (Fallén) .............................72-90

Pachygnatha listeri Sundevall .....................179, 185, 191

Paidiscura pallens (Blackwall) ..............................72-90

Palliduphantes pallidus (P-Cambridge) ...............166, 190

Palloptera usta (Meigen) ....................................... 72-90

Palpares libelluloides (Linnaeus) .......................221, 223

Palpomyia pubescens Kieffer.............................. 72-90

Palpomyia serripes (Meigen) ................................. 72-90

Panolis flammea (Denis \& Schiffermueller) ............72-90

Papestria biren (Goeze) ......................................... 72-90

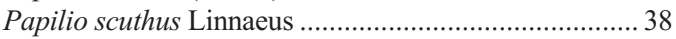

Paracrocera orbicula (Fabricius) ......................... 72-90

Paraleius leontonycha Travé............................... 72-90

Pardosa adustella (Roewer) ...................................... 116

Pardosa algens (Kulczynski) ...................................... 116

Pardosa amentata (Clerck)................................ 174, 191

Pardosa fulvipes (Collett)................................. 174, 191

Pardosa hyperborea (Thorell) .....

174,191

Pardosa jeniseica Eskov \& Marusik.............113, 115, 117

Pardosa lapponica (Thorell) ....................................... 116

Pardosa lugubris (Walckenaer)................72-90, 174, 191

Pardosa lyrata (Odenwall).........................113, 116-117

Pardosa paludicola (Clerck) ............................... 174, 191

Pardosa palustris (Linnaeus) ........................ 174-175, 191

Pardosa pernix (Thorell)............................................. 175

Pardosa plumipes (Thorell)....................................... 115

Pardosa pullata (Clerck).....

175,191

Pardosa ricta (Odenwall)

Pardosa riparia (Koch)

115

Pardosa schenkeli Lessert

$175,185,191$

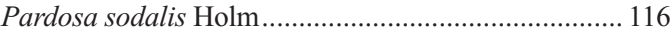

Pardosa sphagnicola (Dahl).............................175, 191

Pardosa tarsalis (Thorell) .................................... 174-175

Pardosa tesquorum (Odenwall) ........................... 115-116

Pegomya winthemi (Meigen)................................... 72-90

Pelecopsis dorniana Heimer ....................................... 115

Pelecopsis elongata (Wider)...............................166, 190

Pelecopsis mengei (Simon) .................................166, 190

Pellenes ignifrons (Grube) ................................. 116-117

Pellenes lapponicus (Sundevall)......................... 178, 191

Pellenes limbatus Kulczynski ...........................113, 117

Peltis grossa (Linnaeus).............................................. 6-22

Pentanota meuseli Bernhauer................................. 6-22
Pentaphyllus testaceus (Hellwig) .................. 6-22

Peponocranium praeceps Miller ..........................166, 190

Peribaea setinervis (Thomson)................................ 72-90

Phaonia incana (Wiedermann).............................. 72-90

Phaonia meigeni Pont ......................................... 72-90

Phauloppia coineaui Travé ................................... 72-90

Phauloppia lucorum (Koch)............................... 72-90

Phauloppia saxicola Travé..................................... 72-90

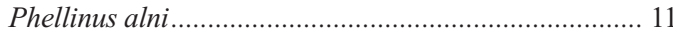

Phellinus cinereus ...................................... 7, 11-12

Phellinus igniarius ......................................... 7, 11-12

Phellinus nigricans..................................... 7, 11-12

Phellinus punctatus.................................................... 12

Phellinus robustus......................................................... 12

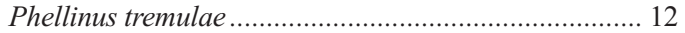

Philodromus alascensis Keyserling ............................... 116

Philodromus aureolus (Clerck) ........................ 72-90, 115

Philodromus cespitum (Walckenaer) ..................... 72-90

Philodromus collinus Koch .................................. 72-90

Philodromus fuscomarginatus (DeGeer) ..........115, 72-90

Philodromus histrio (Latreille) ................................... 115

Philodromus margaritatus (Clerck) ........................ 72-90

Philonthus carbonarius (Gravenhorst) ..................... 6-22

Philonthus fimetarius (Gravenhorst)....................... 6-22

Philonthus subuliformis (Gravenhorst) ..................... 6-22

Philonthus succicola Thomson.................................. 6-22

Phloeocharis subtilissima Mannerheim ........6-22, 57-59,

.72-90

Phloeonomus lapponicus (Zetterstedt).................... $72-90$
Phloeonomus pusillus (Gravenhorst)..................... 6-22

Phloeotribus spinulosus (Rey)...........................198-210

Phoenicocoris obscurellus (Fallén)........................... 72-90

Phora holoserica Schmitz...................................... 72-90

Phora obscura (Zetterstedt) ................................. 72-90

Phragmites ............................................. 166, 170-176

Phrurolithus festivus (Koch)..............................153, 188

Phryganophilus auritus Motschulsky ...................... 6-22

Phylidorea (Paraphylidorea) fulvonervosa (Schummel)47

Phylidorea squalens (Zetterstedt) ...........................72-90

Phyllocnistis labyrinthella (Bjerkander) ................. 72-90

Phyllomyza formicae Schmitz ............................... 72-90

Phyllomyza securicornis ..................................... 72-90

Phyllotetra atra lorestanica Warchalowski..................139

Phyllotreta lorestanica Warchalowski .................138-141

Phyllotreta nigripes (Fabricius)................................... 140

Phyllotreta vittula (Redtenbacher).............................. 140

Phymatura brevicollis (Kraatz) ............................... 6-22

Phytomyza nigrifemur Hering .............................. 72-90

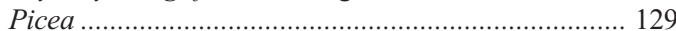

Picea abies ......................... 7, 55, 59, 67, 104, 150, 199

Pinguicula vulgaris ......................................... 124-128

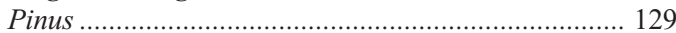

Pinus sylvestris .............. 7, 55, 59, 65-90, 105, 150, 199

Piptoporus betulinus .............................................. 6-22

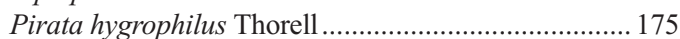

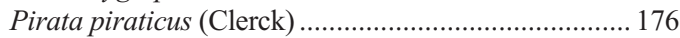

Pirata uliginosus (Thorell) ......................................... 176

Pissodes pini (Linnaeus) ...................................... 72-90

Pissodes piniphilus (Herbst)................................... 72-90

Pityogenes bidentatus (Herbst) .................57-59, 198-210 
Pityogenes chalcographus (Linnaeus).........57-59, 72-90, $198-210$

Pityogenes irkutensis Eggers... $198-210$

Pityogenes quadridens (Hartig) $57-59,198-210$

Pityogenes saalasi Eggers.... $198-210$

Pityogenes trepanatus (Nördlinger)

$198-210$

Pityohyphantes phrygianus (Koch) ..........72-90, 166, 190

Pityophagus ferrugineus (Linnaeus) ............57-59, 72-90

Pityophthorus glabratus Eichhoff....................... 198-210

Pityophthorus lapponicus Stark ........................ 198-210

Pityophthorus lichtensteinii (Ratzeburg)............. 198-210

Pityophthorus micrographus (Linnaeus) ..72-90, 198-210

Pityophthorus morosovi Spessivtseff .................. 198-210

Pityophthorus traegardhi Spessivtseff ................198-210

Pityphilus fasciculatus (DeGeer) ........................... 72-90

Placusa tachyporoides (Waltl)................................. 6-22

Platycladus ...................................................... 129

Platynothrus peltifer (Koch)................................. 72-90

Platypalpus boreoalpinus Frey ............................ 72-90

Platypalpus nigritarsis (Fallén) ............................. 72-90

Plebejus idas (Linnaeus) ............................................. 101

Plectrocnemia conspersa (Curtis)............................ 72-90

Plesiodema pinetella (Zetterstedt) ......................... 72-90

Pleurozium....................................................... 154

Pocadicnemis pumila (Blackwall)....72-90, 166-167, 190

Pocadius ferrugineus (Fabricius).............................. 6-22

Poeciloneta globosa (Wider)...................................... 167

Poeciloneta variegata (Blackwall) ......................167, 190

Pollenia pediculata Macquart ............................... 72-90

Polydrusus undatus (Fabricius) .............................72-90

Polygraphus poligraphus (Linnaeus)................... 198-210

Polygraphus punctifrons Thomson ...................... 198-210

Polygraphus subopacus (Thomson) ....................198-210

Polyommatus amandus (Schneider) ............................ 101

Polyommatus icarus (Rottemburg) ............................... 101

Polyporus alveolarius ................................................ 12

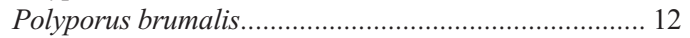

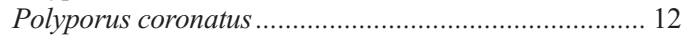

Polyporus melanopus........................................... 12

Polyporus squamosus........................................... 6-22

Populus davidiana ...................................................... 130

Populus tremula................................ 7, 45, 55, 59, 199

Porrhomma campbelli P-Cambridge .................. 167, 190

Porrhomma fagei Miller \& Kratochvil ......................... 167

Porrhomma pallidum Jackson ............................ 167, 190

Porrhomma pygmaeum (Blackwall) .................... 167, 190

Porricondyla fuscostriata Panelius .......................... 72-90

Postia caesia ......................................................... 13

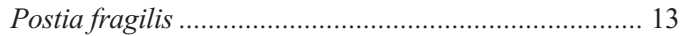

Pristiphora coniceps Lindquist............................... 72-90

Pristiphora pallidiventris Fallén............................. 72-90

Pristiphora pallipes Lepeletier .............................. 72-90

Prolinyphia peltata ............................................... 165

Proteinus atomarius Erichson .................................. 6-22

Proteinus brachypterus (Fabricius) ............................ 6-22

Proteinus macropterus (Gravenhorst)....................... 6-22

Proteinus ovalis Stephens.................................... 6-22

Pseudachorutes subcrassus Tullberg......................72-90

Pseudeuophrys callida (Walckenaer) ...........................178

Pseudeuophrys erratica (Walckenaer) ....................178, 191
Pseudisotoma sensibilis Tullberg........................... 72-90

Pseudobathystomus (Atlantobathystomus) macaronesius sp. n. ....................................225-230

Pseudobathystomus Belokobylskij .....................225-230

Pseudobathystomus funestus (Haliday) ......................2229

Pseudobathystomus macaronesius sp. n. ............225-230

Pseudobathystomus tobiasi (Zaykov)...........225-226, 229

Pseudobathystomus vernalis Belokobylskij................226

Pseudopomyza atrimana (Meigen).......................... 72-90

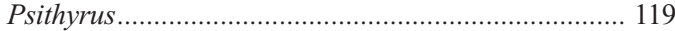

Psylliodes chalcomera (Illiger) ..................................... 140

Psylliodes hyoscyami (Linnaeus)................................ 140

Pteryngium crenatum (Fabricius) ............................ 6-22

Ptinus fur Linnaeus ................................................ 72-90

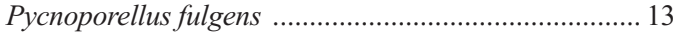

Pycnoporus cinnabarinus ............................................. 13

Pyrgus malvae (Elwes \& Edwards) ............................ 101

Quedius brevicornis Thomson.................................. 6-22

Quedius cruentus (Olivier)...................................... 6-22

Quercus ........................................................... 55, 59

Quercus brandtii...................................................... 219

Quercus cedrorum ................................................... 219

Quercus infectoria ................................................... 219

Quercus liaotungensis........................................... 130

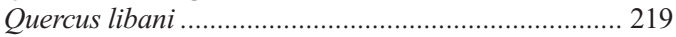

Quercus robur ................................................. 7, 199

Ramonda ringdahli (Villeneuve) ........................... 72-90

Reuterella helvimacula Enderlein............................72-90

Rhadicoleptus alpestris (Kolenati) ........................ 72-90

Rhagonycha atra (Linnaeus) ................................ 72-90

Rhagonycha elongata (Fallen)................................ 72-90

Rhagonycha lignosa (Müller)................................. 72-90

Rhamphomyia (Holoclera) umbripennis Meigen .....72-90

Rhamphomyia (Megcyttarsus) crassirostris (Fallén) 72-90

Rhamphomyia (Pararhamphomyia) angulifera Frey......................................................... 72-90

Rhamphomyia (Pararhamphomyia) caesia Meigen .72-90 Rhamphomyia (Pararhamphomyia) curvula Frey....72-90

Rhamphomyia (Pararhamphomyia) lividiventris

(Zetterstedt).................................................. 72-90

Rhamphomyia (Pararhamphomyia) murina Collin..72-90

Rhamphomyia (Pararhamphomyia) obscuripennis

Meigen

$72-90$

Rhamphomyia (Pararhamphomyia) poplitea ..................

Wahlberg................................................... 72-90

Rhamphomyia (Pararhamphomyia) unguiculata ..............

Frey.......................................................... 72-90

Rhamphomyia (s.str) stigmosa Macquart .................72-90

Rhaphium longicorne (Fallén)............................... 72-90

Rhipidia (Rhipidia) maculata Meigen ........................... 47

Rhipidia (Rhipidia) uniserata Schiner ........................... 47

Rhizophagus bipustulatus (Fabricius) ......................6-22

Rhizophagus dispar (Paykull)....6-22, 57-59, 72-90, 110

Rhizophagus parvulus (Paykull)............................. 6-22

Rhynchites cupreus (Linnaeus) ...............................57-59

Rhysipolis Foerster .................................................. 225

Rhyssaloides Belokobylskij ..................................... 225

Rhyssalus Haliday ................................................... 225

Rigidoporus corticola ................................................ 13

Rigidoporus latemarginatus ...................................... 13 
Rigidoporus ulmarius 13

Robertus arundineti (P-Cambridge) 180,192

Robertus lividus (Blackwall). $180,184,192$

Robertus lyrifer Holm 180,192

Robertus scoticus Jackson ...............72-90, 181, 184, 192

Robinia pseudoacacia ............................................... 50

Rogas (Colastes) funestus Haliday ................................ 225

Ropalodontus strandi (Lohse) ................................. 6-22

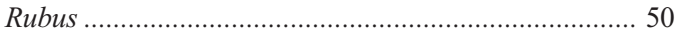

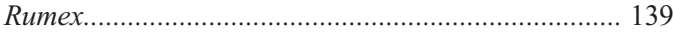

Salix .................................................... 7, 55, 59, 171

Salticus cingulatus (Panzer)................................ 178, 191

Sarracenia purpurea .............................................. 124

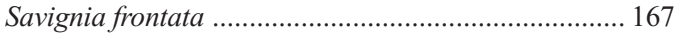

Savignia frontata Blackwall ............................. 167, 190

Savignia nenilini Marusik.................................... 116-117

Scaphidema metallicum (Fabricius).......................... 6-22

Scaphidium quadrimaculatum Olivier ..................... 6-22

Scaphisoma agaricinum (Linnaeus) ......................... 6-22

Scaphisoma assimile Erichson ................................. 6-22

Scaphisoma balcanicum Tamanini ............................ 6-22

Scaphisoma boleti (Panzer) ....................................... 6-22

Scaphisoma boreale Lundblad ............................... 6-22

Scaphisoma inopinatum Löbl ................................. 6-22

Scaphisoma subalpinum Reitter ............................. 6-22

Scaptomyza teinoptera Hackman ........................... 72-90

Scaptosciara Edwards................................................ 107

Scatopsciara atomaria (Zetterstedt) ........................72-90

Schizohelea leucopeza (Meigen) ........................... 72-90

Schizopora paradoxa .............................................. 10

Sciodrepoides fumatus (Spence)................................ 6-22

Sciodrepoides watsoni (Spence) ............................... 6-22

Sciophila bicuspidata Zaitzev ..................................... 196

Sciophila geniculata (Zetterstedt) .......................193-197

Sciophila longua Garrett...................................... 193-197

Sciophila nitens (Winnertz) ................................ 193-197

Sciphila subbicuspidata Zaitzev \& Økland..................196

Scolytus carpini (Ratzeburg) ............................... 198-210

Scolytus intricatus (Ratzeburg) ........................... 198-210

Scolytus laevis Chapuis ...................................... 198-210

Scolytus mali (Bechstein) ..................................... 198-210

Scolytus morawitzi Semenov .............................. 198-210

Scolytus multistriatus (Marsham) ....................... 198-210

Scolytus ratzeburgi Janson ................................... 198-210

Scolytus rugulosus (Ratzeburg) ........................... 198-210

Scolytus scolytus (Fabricius)................................ 198-210

Scolytus triarmatus (Eggers) ............................... 198-210

Scotinotylus evansi (P-Cambridge) ......................167, 190

Scymnus suturalis Thunberg.................................. 72-90

Sedum acre .................................................... 161

Segestria senoculata (Linnaeus) ............................. 72-90

Selatosomus impressus (Fabricius) ......................... 72-90

Semljicola faustus (P-Cambridge)................167-168, 190

Semljicola latus (Holm)....................................... 168, 190

Senecio jacobeae ............................................... 140

Sepedophilus bipustulatus (Gravenhorst).................. 6-22

Sepedophilus littoreus (Linnaeus)............................. 6-22

Sepedophilus marshami (Stephens) ......................... 6-22

Sepedophilus pedicularius (Gravenhorst) ................. 6-22

Sepedophilus testaceus (Fabricius) ........................... 6-22
Sericoderus lateralis (Gyllenhal)........................... 6-22

Sericus brunneus (Linnaeus) ................................... 72-90

Serromyia femorata (Meigen) ................................ 72-90

Sibianor aurocinctus (Ohlert) ......................178-179, 191

Silometopoides sphagnicola Eskov \& Marusik ....116-117

Silometopus incurvatus (P-Cambridge)................168, 190

Silometopus reussi (Thorell) ................................ 168, 190

Simulium paramorsitans Rubtsov .............................. 127

Simulium truncatum (Lundström) ............................... 127

Simulium vernum Macquart ...................................... 127

Singa sanguinea ..................................................... 152

Sisicus apertus (Holm) ..................................... 168, 190

Sitticus caricis (Westring)................................. 179, 191

Sminthurinus aureus (Lubbock) ........................... 72-90

Sminthurinus flammeolus Gisin ........................... 72-90

Solanum tuberosum ................................................. 50

Solter ledereri Navás.......................................... 221, 223

Sorbus aucuparia ......................................... 55, 59, 67

Sorbus pauhuashanensis .......................................... 130

Speudotettix subfusculus (Fallén) ...........................72-90

Sphaeriestes castaneus (Panzer) ............................. 72-90

Sphagnum ........... 150, 159, 162-164, 167-168, 173-177,

179-183

Sphagnum fuscum ......................................... 173-175

Sphinx pinastri Linnaeus ....................................... 72-90

Spilogona cf. albisquama (Ringdahl) .....................72-90

Spilogona contractifrons (Zetterstedt) ....................72-90

Spilogona sororcula (Zetterstedt) .......................... 72-90

Spongipellis spumea ............................................... 13

Steatoda bipunctata (Linnaeus) .........................181, 192

Steatoda phalerata (Panzer) ............................... 181, 192

Steatodea bipunctata (Linnaeus) ............................ 72-90

Stegana cf. coleoptrata Scopoli............................. 72-90

Steganacarus magnus (Nicolet)............................ 72-90

Stegopterna trigonium (Lundström) ........................... 127

Stemonyphantes lineatus (Linnaeus) .....................168, 190

Stenopsocus lachlani Kolbe.................................. 72-90

Strophosoma capitatum Dejean .............................. 72-90

Succisa pratensis......................................... 232, 237

Sulcacis affinis (Gyllenhal) .................................... 6-22

Sulcacis bidentulus (Rosenhauer) .............................. 6-22

Sulcacis fronticornis (Panzer) ...................6-22, 102-112

Synchita humeralis (Fabricius) ............................ 57-59

Syrphus cryptarum Fabricius....................................... 123

Tachypeza nubila (Meigen) .................................... 72-90

Taeniothrips picipes (Zetterstedt) .......................... 72-90

Tallusia experta (P-Cambridge) ...................168, 184, 190

Tapinocyba insecta (Koch) .............................. 168, 190

Tapinocyba pallens (P-Cambridge)..............168-169, 190

Tapinopa longidens (Wider) ................................ 169, 190

Tarentula aculeata .................................................. 173

Tarentula inquilina .................................................... 173

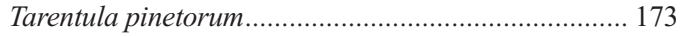

Tarentula pulverulenta ............................................ 173

Tectocepheus velatus (Michael) ............................. 72-90

Tenuiphantes alacris (Blackwall) .....72-90, 169, 184, 190

Tenuiphantes cristatus (Menge) ...........................169, 190

Tenuiphantes mengei (Kulczynski) ....................169, 190

Tenuiphantes tenebricola (Wider) ................169-170, 190

Tetracanthella fjellbergi Deharveng ........................ 72-90 
Tetragnatha dearmata Thorell.

115

Tetragnatha extensa (Linnaeus)

115,117

Tetragnatha montana Simon

72-90

Tetragnatha pinicola Koch.

115

Tetramorium caespitum Linnaeus

213-217

Thalycra fervida (Olivier)

$72-90$

Thanasimus femoralis (Zetterstedt) ....................... 72-90

Thanatus albomaculatus Kulczynski.............................116

Thanatus arcticus Thorell...

116

Thanatus formicinus (Clerck)

177,191

Thanatus striatus Koch.

116-117

Thaumatomyia notata (Meigen)

.72-90

Thaumatomyia trifasciata (Zetterstedt)................... 72-90

Theonoe minutissima (Cambridge) ........................72-90

Thera obeliscata (Hübner) .

Theridion impressum Koch

72-90

Theridion mystaceum Koch

$113,115,117$

181,192

Theridion neglectum Wiehle

181

Theridion notatum (Linnaeus)

181

Theridion pictum (Walckenaer)

115

Theridion pinastri Koch.

72-90

Theridion sibiricum Marusik

115

Theridion sisyphium (Clerck). 72-90, 181, 192

Theridion tinctum (Walckenaer)

$72-90$

Theridion varians Hahn

$72-90$

Thricops cunctans (Meigen)

$72-90$

Thricops innocuus (Zetterstedt)

$72-90$

Thricops lividiventris (Zetterstedt).....

$72-90$

Thricops semicinereus (Wiedemann).....

.72-90

Thrips flavus Schrank

$72-90$

Thrips pini (Uzel).

$72-90$

Thymalus oblongus Reitter.

6-22

Thymelicus lineola (Ochsenheimer) ............................ 101

Thyreosthenius biovatus (P-Cambridge) ..............170, 190

Thyreosthenius parasiticus (Westring) .................170, 190

Tibellus asiaticus Kulczynski ..................................... 115

Tibellus oblongus (Walckenaer).........................177, 191

Tibioploides arcuatus (Tullgren)......................... 170, 190

Tibioplus arcuatus................................................. 170

Tibioplus diversus (Koch)................................ 170, 190

Tilia cordata .......................................... 7, 55, 59, 199

Tilia mandshurica ................................................. 130

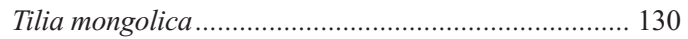

Tillus elongatus (Linnaeus) ..................................... 6-22

Tipula (Lunatipula) circumdata Siebke ..................45, 47

Tipula (Lunatipula) limitata Schummel..........................47

Tipula (Pterelachisus) irrorata Macquart...........47, 72-90

Tipula (Pterelachisus) wahlgreni Lackschewitz.......45, 47

Tipula (Savtshenkia) limbata Zetterstedt........................47

Tipula (Savtshenkia) signata Staeger................................ 47

Tipula (Schummelia) variicornis Schummel...................47

Tipula (Tipula) paludosa Meigen ................................... 47

Tipula (Vestiplex) nubeculosa Meigen ........................... 47

Tipula (Vestiplex) scripta Meigen .....................47, 72-90

Tiso vagans (Blackwall) ..................................... 170, 190

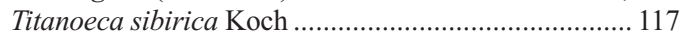

Tobiason Belokobylskij ............................................. 225

Tomicus minor (Hartig)..........................57-59, 198-210

Tomicus piniperda (Linnaeus) ............................. 198-210

Tomoxia bucephala Costa ...................................... 6-22
Trachynella nudipalpis.......................................... 172

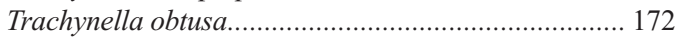

Tradescantia .............................................................. 51

Trametes cervina....................................................... 13

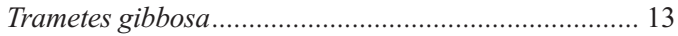

Trametes hirsuta ............................................................. 13

Trametes ochracea ......................................................... 14

Trametes pubescens ............................................... 7, 14

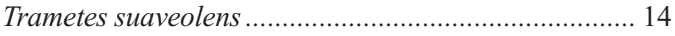

Trametes velutina ............................................. 7, 14

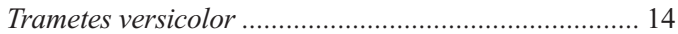

Tricca alpigena (Doleschall) ....................................... 116

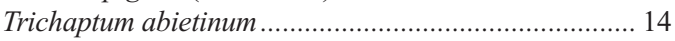

Trichaptum biforme ................................................... 14

Trichaptum fuscoviolaceum .................................... 14

Trichaptum pargamenum ........................................ 14

Trichina bilobata Collin ........................................ 72-90

Trichina clavipes Meigen ........................................ 72-90

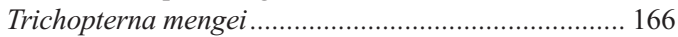

Tricimba cincta (Meigen) ..................................... 72-90

Tricyphona s.str. unicolor (Schummel) ...................72-90

Triogma trisulcata (Schummel) ................................... 47

Triphleba opaca (Meigen)..................................... 72-90

Triphyllus bicolor (Fabricius) ................................ 6-22

Triplax russica (Linnaeus)...................................... 6-22

Tritoma bipustulata Fabricius.................................. 6-22

Tritoma subbasalis (Reitter) .................................... 6-22

Trixa caerulescens Meigen..................................... 72-90

Trochosa ruricola (DeGeer) .............................. 176, 191

Trochosa spinipalpis (P-Cambridge)...................176, 191

Trochosa terricola Thorell..................................176, 191

Troxochrota scabra Kulczynski..........................170, 190

Troxochrus nasutus Schenkel ............................ 170, 190

Troxochrus scabriculus (Westring)...............170, 184, 190

Trypodendron domesticum (Linnaeus)................. 198-210

Trypodendron laeve Eggers ............................... 198-210

Trypodendron lineatum (Olivier) ........................ 198-210

Trypodendron signatum (Fabricius) ..................... 198-210

Trypophloeus alni (Lindemann)......................... 198-210

Trypophloeus asperatus (Gyllenhal) ...................198-210

Trypophloeus bispinulus Eggers ........................... 198-210

Trypophloeus discedens Palm............................. 198-210

Tyromyces chioneus .................................................. 14

Ula bolitophila Loew.............................. 45, 47, 107-108

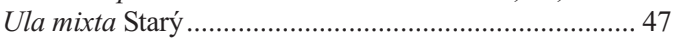

Ula sylvatica (Meigen)................................................ 47

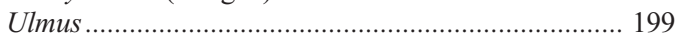

Ulmus glabra .............................................. 199, 206

Ulmus laevis.................................................. 199, 206

Upis ceramboides (Linnaeus) .................................... 6-22

Urtica dioica.............................................................. 50

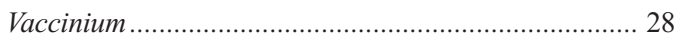

Vaccinium myrtillus ............................... 151, 165, 170

Valenzuela burmeisteri (Brauer) ............................. 72-90

Valenzuela despaxi (Badonnel) ................................. 72-90

Valenzuela gynapterus (Tetens) ............................. 72-90

Variimorda villosa (Schrank)................................... 6-22

Velleius dilatatus (Fabricius) .................................. 6-22

Vespula rufa (Linnaeus) ......................................... 72-90

Wagaicis wagai (Wankowicz) .................................. 6-22 
Walckenaeria antica (Wider)........170-171, 184-185, 190

Walckenaeria atrotibialis (P-Cambridge).............171, 190

Walckenaeria cucullata (Koch) ..........................171, 190

Walckenaeria cuspidata Blackwall ..............171, 184, 190

Walckenaeria dysderoides (Wider)...........72-90, 171, 190

Walckenaeria holmi Millidge............................. 171-172

Walckenaeria karpinskii (P-Cambridge) .............116-117, $171-172,190$

Walckenaeria melanocephala P-Cambridge . .. 171

Walckenaeria nudipalpis (Westring) 172,191

Walckenaeria obtusa Blackwall 172,191

Walckenaeria unicornis P-Cambridge. 172,191

Walckenaeria vigilax (Blackwall) 172,191

Wanachia triguttata (Gyllenhal) .. 6-22

Wideria antica. 170-171

Wideria cucullata 171

Wideria fugax (P-Cambridge) 171

Wideria melanocephala (P-Cambridge)

Wiehlenarius boreus Eskov 171

Winnertzia nigripennis Kieffer $13,116-117$

Winnertzia Rondani 107

Xenillus tegeocranus (Hermann) 107

Xenylla boerneri Axelson .72-90

Xenylla brevicauda Tullberg 72-90

Xenylla maritima Tullberg

72-90

Xerolycosa nemoralis (Westring)

72-90

Xyela julii (Brebisson)

177,191

Xylaplothrips fuliginiosus Schille 72-90

Xyleborus cryptographus (Ratzeburg) ..72-90

Xyleborus dispar (Fabricius)

198-210

Xyleborus monographus (Fabricius)
Xylechinus pilosus (Ratzeburg)

Xysticus audax (Schrank)....

$198-210$

Xysticus austrosibiricus Logunov \& Marusik

Xysticus baltistanus (Caporiacco).

Xysticus bifasciatus Koch

Xysticus britcheri Gertsch

Xysticus cristatus (Clerck)....

Xysticus emertoni Keyserling

Xysticus lineatus (Westring)

Xysticus luctuosus (Blackwall).

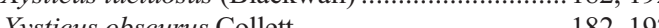

Xysticus sabulosus (Hahn)............................... 182, 192

Xysticus ulmi (Hahn)

183,192

Zelotes clivicola (Koch) .................................... 155, 188

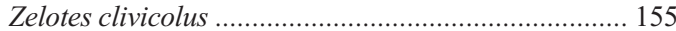

Zelotes fratris (Chamberlin) .........................113, 115, 117

Zelotes latreillei (Simon) ............................. 155, 184, 188

Zelotes praeficus.... 153

Zelotes pratensis (Koch)................................... 155, 189

Zelotes pusillus ................................................... 153-154

Zelotes subterraneus (Koch)............................... 155, 189

Zilora elongata Sahlberg......................................... 6-22

Zora nemoralis (Blackwall)................................183, 192

Zora spinimana (Sundevall) .......................183-184, 192

Zornella cultrigera (Koch) ................................ 172, 190

Zygaena filipendulae Linnaeus.............................91-101

Zygaena lonicerae Scheven..................................91-101

Zygaena loti (Denis \& Schiffermüller) ........................... 98

Zygaena osterodensis Reiss .................................91-101

Zygaena viciae Denis \& Schiffermüller.................91-101

Zygiella stroemi (Thorell).................................... 72-90 